\title{
On the Localization of Topological Invariants
}

\author{
I. M. Gelfand ${ }^{1}$ and B. L. Tsygan ${ }^{2}$ \\ 1 Moscow State University, Moscow, USSR and Rutgers University, New Brunswick, NJ 08903, \\ USA \\ 2 Penn State University, University Park, PA 16802, USA
}

Received September 10, 1990; in revised form March 14, 1991

\begin{abstract}
The approach of formal differential geometry to the topological invariants which can be localized is developed. The universal space and universal characteristic forms are constructed. They give rise to primary and secondary characteristic forms.
\end{abstract}

\section{Introduction}

This paper is a continuation of the works [9-13] and develops the approach of the formal differential geometry. This approach asserts the following. First of all, many of the important topological invariants of a manifold may be localized, i.e., they may be obtained in the following way. Take a finite set of fields $\Gamma_{1}, \ldots, \Gamma_{n}$ on a smooth manifold; by a field we mean a differential geometric object having the prescribed transformation law under the action of the certain transformation group (for example, Riemannian metrics, connections in the given vector bundle, automorphisms of the bundle, various tensors etc.). Construct from the components of these fields and their derivatives some differential form in such a way that it should be covariant under the group of coordinate changes. The space of these forms with the covariance condition has the natural grading and differential. Indeed, the de Rham differential of such a form is again a form constructed covariantly from the fields and their derivatives. So we obtain the cohomological complex which we denote by $\Omega^{*}\left(\Gamma_{1}, \ldots, \Gamma_{n}\right)$. For example, if we have one field $\Gamma=\left(g_{i k}\right)$ which is the Riemannian metric, then the form $\sum_{\alpha, \beta} R_{\alpha \beta} R_{\beta \alpha}$, where $R$ is the curvature tensor is an example of a cocycle lying in $\Omega^{4}(\Gamma)$. Let us maintain that $\sum R_{\alpha \beta} R_{\beta \alpha}$ depending on $g_{i k}$ and their derivatives is regarded as one cocycle - though, of course, it yields a four-form on any Riemannian manifold. The natural question arises, to find the cohomology of the complex $\Omega^{*}\left(\Gamma_{1}, \ldots, \Gamma_{n}\right)$. This cohomology is extremely important. Any cohomology class provides a non-trivial way of constructing covariantly (functorially) a closed form starting from the set of fields 
of a given nature. (By the trivial way of constructing we mean the following: construct covariantly any form, and then take its differential.) For example, consider the complex $\Omega^{*}\left(g_{i k}\right)$, where $\left(g_{i k}\right)$ is the Riemannian metric. By the suggestion of the first author, the cohomology of this complex had been computed in the middle of the 50's by A. A. Abramov. He had proved that the cohomology ring of the complex $\Omega^{*}\left(g_{i k}\right)$ is the free commutative algebra generated by the Pontryagin classes.

Let $\omega$ be a cocycle of the complex $\Omega^{*}\left(\Gamma_{1}, \ldots, \Gamma_{n}\right)$. Consider a smooth manifold $Y$ together with the two different sets of fields $\left(\Gamma_{1}^{\prime}, \ldots, \Gamma_{n}^{\prime}\right)$ and $\left(\Gamma_{1}^{\prime \prime}, \ldots, \Gamma_{n}^{\prime \prime}\right)$ of a given type. The cocycle $\omega$ allows the construction of two closed forms $\omega\left(\Gamma_{1}^{\prime}, \ldots, \Gamma_{n}^{\prime}\right)$ and $\omega\left(\Gamma_{1}^{\prime \prime}, \ldots, \Gamma_{n}^{\prime}\right)$ on $Y$. It is not hard to show that the difference $\omega\left(\Gamma_{1}^{\prime}, \ldots, \Gamma_{n}^{\prime}\right)-\omega\left(\Gamma_{1}^{\prime \prime}, \ldots, \Gamma_{n}^{\prime \prime}\right)$ is exact if the sets $\left(\Gamma_{1}^{\prime}, \ldots, \Gamma_{n}^{\prime}\right)$ and $\left(\Gamma_{1}^{\prime \prime}, \ldots, \Gamma_{n}^{\prime \prime}\right)$ belong to the same homotopy type. Thus, the cohomology class of the form $\omega\left(\Gamma_{1}^{\prime}, \ldots, \Gamma_{n}^{\prime}\right)$ provides the topological invariant of the manifold $Y$ and the homotopy class of $n$-tuples $\left(\Gamma_{1}^{\prime}, \ldots, \Gamma_{n}^{\prime}\right)$.

This idea has some resemblance with the idea of ghosts in theoretical physics. Indeed, the topological invariant does not depend on a choice of the concrete fields, but using these fields which play the role of ghosts is very important because it allows to localize the invariant.

The topological invariants which may be obtained by the described procedure are: the characteristic classes of the vector bundles (i.e., the Chern character of $K^{0}$ ), the characteristic classes of the automorphisms of vector bundles (i.e., the Chern character of $K^{1}$ ), in particular, the index of the pseudodifferential elliptic operators, the Chern character of higher $K$ theory, the determinants of certain elliptic operators (Burglelea, Friedlander, Kappeller [2]). Note that for such an invariant one may start the procedure of localization from different sets of fields $\Gamma_{i}^{\prime} s$. For example, the characteristic classes of vector bundles may be obtained as the cohomology classes of the closed forms constructed from one of the following data: a metric in a bundle, a connection, a superconnection (in sense of Quillen, [16]), a smooth map putting in correspondence to any point of the manifold a formal coordinate system in the bundle at this point, etc. One may also consider all the possible closed differential forms which may be constructed covariantly from the components of the two connections in a bundle. In all these cases, the set of invariants which may be obtained from this procedure (i.e., the cohomology of the complex $\Omega^{*}$ ) is probably the same - the Chern (or Pontryagin) classes. Nevertheless, the local expressions themselves, and also the corresponding secondary characteristic classes (see below), are of great interest for all these cases. Similarly, the Chern character for $K^{1}$ may be obtained by the procedure described above starting from the following data: a function on a manifold with values in $G L_{n}$ (i.e., an automorphism of the trivial vector bundle), an automorphism of a vector bundle and a connection in this bundle, a superconnection of Quillen, etc.

We have already mentioned above that for a cocycle $\omega$ of the complex $\Omega^{*}\left(\Gamma_{1}, \ldots, \Gamma_{n}\right)$ and for the two different choices of the fields $\Gamma_{1}^{\prime}, \ldots, \Gamma_{n}^{\prime}$ and $\Gamma_{1}^{\prime \prime}, \ldots, \Gamma_{n}^{\prime \prime}$ the difference $\omega\left(\Gamma_{1}^{\prime}, \ldots, \Gamma_{n}^{\prime}\right)-\omega\left(\Gamma_{1}^{\prime \prime}, \ldots, \Gamma_{n}^{\prime \prime}\right)$ is exact if $\left(\Gamma_{1}^{\prime}, \ldots, \Gamma_{n}^{\prime}\right)$ and $\left(\Gamma_{1}^{\prime \prime}, \ldots, \Gamma_{n}^{\prime \prime}\right)$ are in the same homotopy class. Moreover, one may construct canonically the differential form $\eta$ such that $\omega\left(\Gamma_{1}^{\prime}, \ldots, \Gamma_{n}^{\prime}\right)-\omega\left(\Gamma_{1}^{\prime \prime}, \ldots, \Gamma_{n}^{\prime \prime}\right)=d \eta$. This form $\eta$ may depend only on $\Gamma_{1}^{\prime}, \ldots, \Gamma_{n}^{\prime}, \Gamma_{1}^{\prime \prime}, \ldots, \Gamma_{n}^{\prime \prime}$ (in the case of metrics, connections or Quillen superconnections in a finite dimensional bundle) but may 
also depend on a path connecting $\left(\Gamma_{1}^{\prime}, \ldots, \Gamma_{n}^{\prime}\right)$ and $\left(\Gamma_{1}^{\prime \prime}, \ldots, \Gamma_{n}^{\prime \prime}\right)$ (in the case of automorphism of a vector bundle or Quillen superconnections in an infinite dimensional bundle). The form $\eta$ is called the secondary form.

One may construct also the higher secondary forms. Having three different sets of data $\left(\Gamma_{1}^{\prime}, \ldots, \Gamma_{n}^{\prime}\right),\left(\Gamma_{1}^{\prime \prime}, \ldots, \Gamma_{n}^{\prime \prime}\right)$ and $\left(\Gamma_{1}^{\prime \prime \prime}, \ldots, \Gamma_{n}^{\prime \prime \prime}\right)$ one may construct the form $\zeta$ (possibly depending on some supplementary data) such that $d \zeta=$ $\eta\left(\Gamma_{1}^{\prime}, \ldots, \Gamma_{n}^{\prime} ; \Gamma_{1}^{\prime \prime}, \ldots, \Gamma_{n}^{\prime \prime}\right)-\eta\left(\Gamma_{1}^{\prime}, \ldots, \Gamma_{n}^{\prime} ; \Gamma_{1}^{\prime \prime \prime}, \ldots, \Gamma_{n}^{\prime \prime \prime}\right)+\eta\left(\Gamma_{1}^{\prime \prime}, \ldots, \Gamma_{n}^{\prime \prime} ; \Gamma_{1}^{\prime \prime \prime}, \ldots, \Gamma_{n}^{\prime \prime \prime}\right)$ and so on.

All these secondary forms are very important. There is a nontrivial interplay between characteristic classes and secondary forms for various sets of initial fields $\Gamma_{i}^{\prime} s$ giving rise to various topological invariants; also, the secondary forms give rise to the functional integrals which may be important for the field theory. For example, the first secondary form for the cocycle $\sum R_{\alpha \beta} R_{\beta \alpha}$ discussed above is known as the famous Chern-Simons form.

The secondary forms for the Pontryagin classes had been introduced by Gabrielov, Gelfand and Losik in [10], the Chern-Simons form had been introduced independently in [4].

In this paper, continuing the work [10], we show that all these secondary forms and the relations between them may be obtained from one universal construction. Assume that the topological invariant under consideration may be obtained as described above with certain differential geometric objects, or fields (i.e., functions, forms, automorphisms, connections, etc.). We introduce a new differential manifold whose points are jets of such fields. This manifold is infinite dimensional and has the natural structure of a fibre bundle over the original manifold (one may associate to a jet at a point this point itself). There are two canonical structures on such a manifold of jets.

On the one hand, on the space of jets of fields of a given nature there is the universal field of the same nature (i.e., the natural function on the space of jets of functions, etc.). This field is the result of evaluation: we put in correspondence to a jet of a field its value at the origin. On the other hand, the space of jets of fields (which is a fibre bundle, as mentioned above) carries the natural flat connection, i.e., the integrable system of horizontal tangent subspaces which is covariant with respect to the natural group of transformations. This system provides the local structure of direct product and thus the decomposition of the de Rham complex of the manifold of jets. So, the de Rham differential on this manifold is the sum of the two differentials - the horizontal one and the vertical one.

Furthermore, having the universal space of jets with the universal field on it, one may construct the canonical form (see above) according to this field. Since the de Rham complex admits the decomposition, this universal form is represented by the collection of its homogeneous components. These components are the universal secondary classes. Since the universal form is closed, we obtain several identities concerning these classes. Among them are the invariance of the characteristic form under the choice of fields and higher relations among secondary classes.

We consider the following examples:

1. The field is the connection in a bundle over a manifold (maybe it would be more correct to include the bundle itself into the set of fields); the form constructed 
from these fields is the invariant polynomial of the curvature; the corresponding topological invariants are the Chern classes. In this case, our approach was developed in [10], it was applied to the combinatorial construction of the first Pontryagin class. The first of the secondary forms was introduced independently by Chern and Simons [4]. We recall this construction in Sects. 1,4.

2. The fields are the connection in a bundle and the fibrewise automorphism of this bundle. The topological invariant is the Chern character of the corresponding element of $K^{1}$. In Sect. 2 we define a corresponding characteristic form; in Sect. 5 we develop the approach outlined above.

3 . The field is the Quillen superconnection on a $Z_{2}$-graded vector bundle; the form is the Quillen character form ([16]); the topological invariant is the Chern character of the bundle or the Chern character of the index of the family. We describe the corresponding construction in Sects. 3,6.

Note that a secondary form for one problem may be a primary form for another problem. We discuss this phenomenon in the last two paragraphs. In Sect. 8, we assume that a connection $\nabla$ in a vector bundle and an automorphism $\sigma$ of this bundle are given. Then one may construct another connection $\nabla^{\sigma}$ which is the result of the action on $\sigma$ on $\nabla$. Since the characteristic forms are invariant under the coordinate changes, clearly the connections $\nabla$ and $\nabla^{\sigma}$ have the same characteristic forms. Thus, the secondary characteristic forms for the pair of $\nabla$ and $\nabla^{\sigma}$ are closed. We show that these forms are the "primary" forms from Sect. 2 (see the Introduction). This illustrates the well known fact that Chern character for $K^{1}$ is the transgressed Chern character for $K^{0}$. As another example of the same phenomenon, we recall in our language the Connes-Moscovici construction of the Chern character of Fredholm modules [5]. We show that the corresponding Chern character forms are secondary forms for the canonical superconnection on the universal space of jets of superconnections where the underlying manifold is a point. Along the same lines, one may also develop a similar approach to characteristic classes in higher $K$ theory. We will discuss this in our next work. We hope that this approach will be useful in attempts to give a combinatorial description of the index of an elliptic operator. Note also that the secondary forms of the examples above are useful in topological field theory, WZW theory and matrix models $[7,18,19]$.

\section{Chern Classes of the Vector Bundles}

Let $Y$ be a smooth manifold, $n=\operatorname{dim} Y$; let $E$ be a complex vector bundle of rank $N$ over $Y$. One may define the Chern classes of $E$ as follows (cf. [14]). Let $\nabla$ be a connection in $E$. Let $R$ be the curvature of $\nabla$. This is a two-form on $Y$ with values in $\operatorname{End}(E)$.

In coordinates, let $e_{1}(y), \ldots, e_{N}(y)$ be a basis in the fiber $E$ of $E$ which depends smoothly on a point $y$ of an open neighborhood $U$ of $Y$. Then connection $\nabla$ is given by a matrix valued one-form $\omega=\left(\omega_{i j}\right)_{1 \leqq i, j \leqq N}$ on $U$. If $e_{1}^{\prime}(y), \ldots, e_{N}^{\prime}(y)$ is another basis and $e_{i}^{\prime}=\Sigma g_{i j}(y) e_{i}$, then the form $\omega^{\prime}$ corresponding to this basis is given by the gauge transformation:

$$
\omega^{\prime}=d g \cdot g^{-1}+g \omega g^{-1}
$$


the curvature form $R$ is given by

$$
R=d \omega+\omega^{2}
$$

It is easy to see that in coordinates corresponding to the basis $e_{1}^{\prime}, \ldots, e_{N}^{\prime}$ the form $R^{\prime}$ is given by

$$
R^{\prime}=g R g^{-1}
$$

Now, let $P$ be a homogeneous polynomial of degree $m$ on the Lie algebra $g l(N, \mathbb{C})$ which is invariant under the adjoint action of $G L(N, \mathbb{C})$. Put

$$
c_{P}(E, \nabla)=P(R)
$$

This is a well defined $2 m$-form on $Y$.

Example 1. Let $X \in g l(N, \mathbb{C}), P(X)=\frac{1}{m !} \operatorname{tr} X^{m}$. The form $c_{P}(E, \nabla)$ is denoted by $\mathrm{ch}_{m}(E, \nabla)$. In coordinates, if $R=\left(R_{i j}\right)^{m !}$

$$
\operatorname{ch}_{m}(E, \nabla)=\frac{1}{m !} \sum_{i_{1}, \ldots, i_{m}} R_{i_{1} i_{2}} \wedge R_{i_{2} i_{3}} \wedge \cdots \wedge R_{i_{m} i_{1}} .
$$

The element $\sum_{m=0}^{\infty} \operatorname{ch}_{m}(E, \nabla)$ of $\Omega_{Y}^{*}$ is called the Chern character form of $\nabla$ and is denoted by $\operatorname{ch}(E, \nabla)$.

Example 2. For any subset $I$ of $\{1, \ldots, N\}$ and for any $X \in g l(N, \mathbb{C})$ denote by $\left|X_{I}\right|$ the minor $\operatorname{det}\left(x_{i j}\right)_{i, j \in I}$. Define a polynomial $P$ by

$$
P(X)=\sum_{\# I=m}\left|X_{I}\right|
$$

The form $c_{P}(E, \nabla)$ is denoted by $c_{m}(E, \nabla)$. In coordinates,

$$
c_{m}(E, \nabla)=\sum_{i_{1}<\cdots<i_{m}} \sum_{\sigma \in S_{m}} \operatorname{sgn} \sigma \cdot R_{i_{1} i_{\sigma(1)}} \wedge \cdots \wedge R_{i_{m} i_{\sigma(n)}} .
$$

Theorem 1. (cf. [14]). For any homogeneous invariant polynomial $P$ on $g l(N, \mathbb{C})$ and for any connection $\nabla$ in the fibre bundle $E$, the form $c_{P}(E, \nabla)$ is closed. For any two connections $\nabla, \nabla^{\prime}$ in $E$, the difference $c_{P}(E, \nabla)-c_{P}\left(E, \nabla^{\prime}\right)$ is exact.

Thus, the cohomology class of the form $c_{P}(E, \nabla)$ does not depend on $\nabla$. This class is denoted by $c_{P}(E) \in H_{\infty}^{2 m}(Y, \mathbb{C})$. If $P$ is as in Example 1 , the class $c_{P}(E)$ is denoted by $\operatorname{ch}_{m}(E)$; the class $\sum_{m=0}^{\infty} \operatorname{ch}_{m}(E)$ is denoted by $\operatorname{ch}(E)$ and is called the Chern character of $E$. If $P$ is as in Example 2, the class $c_{P}(E)$ is denoted by $c_{m}(E)$ and is called the $m^{\text {th }}$ Chern class of $E$.

If $E$ is a real bundle, then, as it is well known, $c_{2 k+1}(E \otimes \mathbb{C})=0$; the class $c_{2 k}(E \otimes \mathbb{C})$ is denoted by $p_{k}(E)$ and called the $k^{\text {th }}$ Pontryagin class of $E$. It is an element of $H^{4 k}(Y, \mathbb{R})$. 


\section{Chern Character for $K^{1}$}

Let $Y$ be a smooth $n$-dimensional manifold and $E$ a complex vector bundle of rank $N$ over $Y$. Let $\sigma \in \Gamma(Y$, Aut $(E))$ be a smooth section of the bundle of fibrewise automorphisms of $E$. In coordinates, if $e_{1}(y), \ldots, e_{N}(y)$ is a smooth basis as in Sect. 1, then $\sigma$ is represented by an invertible matrix $\left(\sigma_{i j}\right)_{1 \leqq i, j \leqq N}$; if $e_{1}^{\prime}(y), \ldots, e_{N}^{\prime}(y)$ is another smooth basis, $e_{i}^{\prime}=\Sigma g_{i j} e_{j}$, then the matrix corresponding to this new basis is given by $\sigma^{\prime}=g \sigma g^{-1}$. As it is well known from [1], an element $\sigma$ determines an element $[\sigma]$ of the group $K^{1}(Y)$ and the elements $\operatorname{ch}_{m}([\sigma])$ of the spaces $H^{2 m-1}(X, \mathbb{C})$. The aim of this section is to give the explicit representation of these elements by the closed forms on $Y$.

First, note that if the bundle $E$ is trivial, $E=Y \times \mathbb{C}^{N}$, the element $\sigma$ is simply a smooth map $Y \rightarrow G L(N, \mathbb{C})$ and the cohomology class of $\operatorname{ch}_{m}[\sigma]$ is given by the closed form (cf. [14]),

$$
\mathrm{ch}_{m}(\sigma)=\frac{(-1)^{m-1}}{2^{m-1}(2 m-1) ! !} \operatorname{tr}\left(d \sigma \cdot \sigma^{-1}\right)^{2 m-1}
$$

to prove an analogous statement in general, we need some auxiliary definitions.

1. The Polynomials $P$. Let $\mathbb{C}\{x, y, z\}$ be the free associative algebra with three generators. Let

$$
\mathscr{A}=\mathbb{C}\{x, y, z\} /\left(x z, z y, y^{2}, z^{2}\right)
$$

be the quotient of $\mathscr{A}$ by the two-sided ideal generated by $x z, z y, y^{2}, z^{2} ;$ let $\mathscr{A}_{0}$ be the vector subspace in $\mathscr{A}$ generated by the elements $a b-b a$, where $a, b, \in \mathscr{A}$. Put $\overline{\mathscr{A}}=\mathscr{A} / \mathscr{A}_{0}$. It is easy to see that any element of $\mathscr{A}$ is congruent modulo $\mathscr{A}_{0}$ to a linear combination of the elements $y, z$ and $x^{i_{1}}(y z)^{j_{1}} \cdots x^{i_{k}}(y z)^{j_{k}}$, i.e., is representable by an element $\beta y+\gamma z+f(x, y z)$, where $f$ is an element of the free algebra $\mathbb{C}\{x, u\}$. Moreover, such an element belongs to $\mathscr{A}_{0}$ iff $\beta=\gamma=0$ and $f$ belongs to the linear subspace of $\mathbb{C}\{x, u\}$ generated by the element $a b-b a, a, b \in \mathbb{C}\{x, u\}$. So, one may put in correspondence to an element $g$ of $\mathscr{A}$ the element $\bar{g}$ of $\mathbb{C}\{x, u\} \mid\langle a b-b a\rangle$.

Put

$$
P_{m}(x, u)=\frac{(-1)^{m-1}}{2^{m-1}(2 m-1) ! !} \overline{(x+y+z)^{m}}, \quad m \geqq 1 .
$$

For example,

$$
\begin{aligned}
& P_{1}(x, u)=x \\
& P_{2}(x, u)=-\frac{1}{6}\left(x^{3}+3 x u\right) \\
& P_{3}(x, u)=\frac{1}{60}\left(x^{5}+5 x^{3} u+5 x u^{2}\right) .
\end{aligned}
$$

2. The Character Forms. Let $\nabla$ be a connection in the bundle $E, R$ its curvature form; let $\nabla_{1}$ be the connection in the bundle $\operatorname{End}(E)$ induced by $\nabla$. In coordinates, if $\nabla$ is represented by a matrix $\omega$ (cf. Sect. 1) and $f \in \Gamma(Y, \operatorname{End}(E))$ is represented by a matrix $\left(f_{i j}\right)$ then $\nabla_{1} f=d f+[\omega, f]$. Let $\sigma$ be as in the beginning of Sect. 2.

Theorem 2.i. For any $m \geqq 1$, the form

$$
\operatorname{ch}_{m}(E, \nabla, \sigma)=\operatorname{tr} P_{m}\left(\nabla_{1} \sigma \cdot \sigma^{-1},(\sigma-1) R\left(1-\sigma^{-1}\right)\right)
$$


is closed. Its cohomology class does not depend on a choice of $\nabla$ and is equal to $\mathrm{ch}_{m}([\sigma])$.

Proof. Let $E^{\prime}$ be another bundle, such that $E \oplus E^{\prime}=Y \times \mathbb{C}^{M}$ for some $M$. Let $e$ be the projection onto $E$ along $E^{\prime}$. Let $\tilde{\sigma}=\sigma_{E} \oplus O$ be the endomorphism of the trivial vector bundle $Y \times \mathbb{C}^{M}$. One has $\operatorname{ch}_{m}([\sigma])=\operatorname{ch}_{m}\left(\left[\sigma_{E} \oplus 1_{E^{\prime}}\right]\right)=\operatorname{ch}_{m}(\tilde{\sigma}+1-e)$. One has also ede $e=(1-e) d e(1-e)=0 ; \quad e \tilde{\sigma}=\tilde{\sigma} e=\tilde{\sigma}$; using these identities we have $d \tilde{\sigma} \cdot \tilde{\sigma}^{-1}=d(e \tilde{\sigma} e+1-e)\left(e \tilde{\sigma}^{-1} e+1-e\right)=e \cdot d \tilde{\sigma} \cdot \tilde{\sigma}^{-1} e+e(\tilde{\sigma}-1) d e(1-e)+$ $(1-e) \operatorname{de}\left(1-\tilde{\sigma}^{-1}\right) e$.

Denote the last three summands in the last sum by $x, y, z$. Consider the connection $e \cdot d \cdot e$ in the bundle $E$ (compare with [4]). It is easy to see that $y z=e(\sigma-1) R\left(1-\sigma^{-1}\right) e$ (because $\left.R=e d e d e\right)$. Using the fact that $\operatorname{tr}(e \cdot A \cdot(1-e))=$ $\operatorname{tr}((1-e) \cdot B \cdot e)=0$ for any $A$ and $B$, we see that for the special case of the connection $\nabla \operatorname{ch}_{m}\left(\sigma_{E} \oplus 1_{E^{\prime}}=\operatorname{ch}_{m}(E, \nabla, \sigma)\right.$. In particular, $\operatorname{ch}_{m}(E, \nabla, \sigma)$ is closed.

Now, it is not hard to show that, at least locally, any connection $\nabla$ can be represented as above: $\nabla=e \cdot d \cdot e$, where $E \oplus E^{\prime}=Y \times \mathbb{C}^{N}$ and $e: E \oplus E^{\prime} \rightarrow E$ is projection. This shows that the forms $\operatorname{ch}_{m}(E, \nabla, \sigma)$ are always closed. From this, and from the results of Sect. 6, it follows that the cohomology class of $\operatorname{ch}_{m}(e, \nabla, \sigma)$ does not depend on $\nabla$ (and on $\sigma$, within a given homotopy class). Thus, this class is always equal to $\mathrm{ch}_{m}([\sigma])$.

Example 1. Assume that the bundle $E$ is trivialized over the open subset $U$ of $Y$ and, moreover, that $\sigma$ is diagonalized over this subset. Then $\nabla$ is represented by the matrix $\omega=\left(\omega_{i j}\right)$ of 1 -forms and $\sigma$ is represented by the diagonal matrix with the diagonal terms $\lambda_{1}(y), \ldots, \lambda_{N}(y)$. Then

$$
\begin{aligned}
& \operatorname{ch}_{1}(E, \nabla, \sigma)=\sum_{j=1}^{N} \frac{d \lambda_{j}}{\lambda_{j}} \\
& \operatorname{ch}_{2}(E, \nabla, \sigma)=\frac{1}{2} d\left(\frac{1}{2} \sum_{i, j} \frac{\left(\lambda_{i}-\lambda_{j}\right)\left(\lambda_{i}-1\right)\left(\lambda_{j}-1\right)}{\lambda_{i} \lambda_{j}} \omega_{i} \omega_{j}+\sum_{i} \frac{d \lambda_{i}}{\lambda_{i}} \frac{\left(\lambda_{i}-1\right)^{2}}{\lambda_{i}} d \omega_{i i}\right) .
\end{aligned}
$$

\section{Chern Character Forms for Superconnections}

In this paragraph we follow Quillen [16]. Let $Y$ be a smooth manifold and $E$ a $\mathbb{Z}_{2}$-graded vector bundle over $Y$. This means that $E=E^{\prime} \oplus E^{\prime \prime}$, where $E^{\prime}, E^{\prime \prime}$ are the vector bundles. The spaces $\Omega_{Y}^{*}(E)$ and $\Omega_{Y}^{*}($ End $E)$ have the natural $\mathbb{Z}_{2}$-gradings: for $\omega \in \Omega_{Y}^{k}$ and $\gamma \in \Gamma(Y, E)$ (respectively $\Gamma(Y$, End $E)$ we put $p(\omega \otimes \gamma)=(-1)^{k+p(\gamma)}$, where $p$ denotes the parity. For $\omega \in \Omega_{Y}^{k}, \omega^{\prime} \in \Omega_{Y}^{l}, \gamma, \gamma^{\prime} \in \Gamma\left(Y_{0} E\right)$ (respectively $\Gamma(Y$, End $E))$ put also $(\omega \otimes \gamma) \cdot\left(\omega^{\prime} \otimes \gamma^{\prime}\right)=(-1)^{l \cdot p(\gamma)}\left(\omega \omega^{\prime} \otimes \gamma \cdot \gamma^{\prime}\right)$; this operation turns $\Omega_{Y}($ End $E)$ to be a $\mathbb{Z}_{2}$-graded algebra and $\Omega_{Y}(E)$ a $\mathbb{Z}_{2}$-graded module over $\Omega_{Y}($ End $E)$.

Let $\nabla$ be an even connection in the bundle $E$. Let $X$ be an odd element of the space $\Gamma(Y$, End $E)$. This element determines the operator of left multiplication in the module $\Omega_{Y}^{*}(E)$. We denote this operator by the same letter $X$. A superconnection in the $\mathbb{Z}_{2}$-bundle $E$ is by definition an operator

$$
\nabla+X: \Omega_{Y}^{*}(E) \rightarrow \Omega_{Y}^{*}(E)
$$


Here $\nabla$ is the covariant derivative. In coordinates, if $y \in Y$ runs over an open subset $U$ on which the bundle $E$ is trivial, if $e_{1}^{\prime}(y), \ldots, e_{N^{\prime}}^{\prime}(y)$ and $e_{1}^{\prime \prime}(y), \ldots, e_{N^{\prime \prime}}^{\prime \prime}, \ldots, e_{N^{\prime \prime}}^{\prime \prime}(y)$ are the bases of $E^{\prime}$ and $E^{\prime \prime}$, then the operator $\nabla$ is given by

$$
\left(\begin{array}{l|l}
d+\omega^{\prime} \mid \\
\hline & d+\omega^{\prime \prime}
\end{array}\right): \Omega_{U}^{*} \otimes\left(\mathbb{C}^{N^{\prime}} \oplus \mathbb{C}^{N^{\prime \prime}}\right) \rightarrow \Omega_{U}^{*+1} \otimes\left(\mathbb{C}^{N^{\prime}} \oplus \mathbb{C}^{N^{\prime \prime}}\right)
$$

and the operator $X$ is given by

$$
\left(\begin{array}{c|c}
0 & X^{\prime \prime} \\
\hline X^{\prime} & 0
\end{array}\right): \Omega_{U}^{*} \otimes\left(\mathbb{C}^{N^{\prime}} \oplus \mathbb{C}^{N^{\prime \prime}}\right) \rightarrow \Omega_{U}^{*} \otimes\left(\mathbb{C}^{N^{\prime}} \oplus \mathbb{C}^{N^{\prime \prime}}\right),
$$

where $X^{\prime}=\left(x_{i k}^{\prime}\right)_{i \leq N^{\prime}, k \leq N^{\prime \prime}}$ and $X^{\prime \prime}=\left(x_{i k}^{\prime \prime}\right)_{k \leqq N^{\prime \prime}, i \leq N^{\prime}}$

It is easy to see [16] that the operator $(\nabla+X)$ is the operator of multiplication by an element $R_{s}=R+\nabla_{1} X+X^{2} \in \Omega_{Y}^{*}(\operatorname{End}(E))$. Here $R=R^{\prime} \oplus R^{\prime \prime}$ is the curvature form of $\nabla ; \nabla_{1}$ is the induced connection in $\operatorname{End}(E)$, as in Sect. 1; in coordinates, $\nabla_{1} X$ is given by the matrix of 1 -forms

$$
\left(\begin{array}{c|c}
0 & d X^{\prime \prime}+\omega^{\prime} X^{\prime \prime}-X^{\prime \prime} \omega^{\prime \prime} \\
\hline d X^{\prime}+\omega^{\prime \prime} X^{\prime}-X^{\prime} w^{\prime} & 0
\end{array}\right)
$$

and $X^{2}$ is given by the matrix of functions

$$
\left(\begin{array}{c|c}
X^{\prime \prime} X^{\prime} & 0 \\
\hline 0 & X^{\prime} X^{\prime \prime}
\end{array}\right)
$$

Now we are ready to recall Quillen's definition of the Chern character form. For an element $x$ of $\Omega_{Y}^{*}(\operatorname{End}(E))$ put $\exp (x)=\sum_{n=0}^{\infty} \frac{1}{n !} x^{n}$; it may be shown (cf. [16]) that this gives a well defined element of $\Omega_{Y}^{*}(\operatorname{End}(E))$.

Let $\varepsilon$ in $\Omega_{Y}^{0}(\operatorname{End}(E))$ act on $E_{+}$by multiplication by one and on $E_{-}$by multiplication by minus one. For any element $\omega$ in $\Omega_{Y}^{*}(\operatorname{End}(E)), \operatorname{put}^{\operatorname{tr}_{s}} \omega=\operatorname{tr} \varepsilon \omega$; this is an element of $\Omega_{Y}^{*}$.

Definition. The Chern character form of the superconnection $\nabla+X$ is the element

$$
\operatorname{ch}(E, \nabla+X)=\operatorname{tr}_{s} \exp \left(R^{2}+\nabla_{1} X+X^{2}\right) .
$$

Theorem 3.1. (cf. [16]). The Chern character form $\operatorname{ch}(E, \nabla+X)$ is closed; for any two superconnections $\nabla_{1}+X_{1}$ and $\nabla_{2}+X_{2}$ the difference $\operatorname{ch}\left(E, \nabla_{1}+X_{1}\right)-$ $\operatorname{ch}\left(E, \nabla_{2}+X_{2}\right)$ is exact.

Example 1. Let $X=0$. Then

$$
\operatorname{ch}(E, \nabla)=\operatorname{ch}\left(E^{\prime}, \nabla^{\prime}\right)-\operatorname{ch}\left(E^{\prime \prime}, \nabla^{\prime \prime}\right),
$$

where the Chern character forms in the right-hand side are those of Sect. 1.

Example 2. Assume that $E$ is not a finite dimensional but rather a Hilbert vector bundle over $Y$, assume that $X_{0}$ is Fredholm and skew self adjoint. Thus, it is of the form $\left(\frac{0}{-X_{0}^{*}} \mid \frac{X_{0}}{0}\right)$; if $Y$ is the point, then

$$
\operatorname{ch}\left(0+X_{0}\right)=e^{-X_{0} X_{0}^{*}}-e^{-X_{0}^{*} X_{0}}=\operatorname{index} X_{0}
$$


(by the McKean-Singer formula) If $Y$ is not a point the Chern character form may be defined under some restrictions on $\nabla, X$; its cohomology class is the Chern character of the index of the family $X_{0}$ lying in $K^{0}(Y)$.

Let us give more explicit formulas for the Chern character forms. One has

$$
\operatorname{ch}(E, \nabla+X)=\sum_{n=0}^{\infty} \int_{\substack{t_{0}, \ldots, t_{n} \geqq 0 \\ \sum t_{i}=1}} \operatorname{tr}_{s}\left(e^{t_{0} X^{2}} \times\left(R+\nabla_{1} X\right) e^{t_{1} X^{2}}\left(R+\nabla_{1} X\right) \cdots e^{t_{n} X^{2}} d t_{1} \cdots d t_{n} .\right.
$$

In coordinates,

$$
\begin{aligned}
& \operatorname{ch}(E, \nabla+X)=\sum_{n=0}^{\infty} \int_{\substack{t_{0}, \ldots, t_{n} \geqq 0 \\
\sum t_{i}=1}} \operatorname{tr}\left(\begin{array}{cc}
1 & 0 \\
0 & -1
\end{array}\right)\left(\begin{array}{cc|c}
e^{t_{0} X^{\prime \prime} X^{\prime}} & 0 \\
\hline 0 & e^{t_{0} X^{\prime} X^{\prime \prime}}
\end{array}\right) \\
& \cdot\left(\begin{array}{c|c}
0 & d X^{\prime \prime}+\omega^{\prime} X^{\prime \prime}-X^{\prime \prime} \omega^{\prime} \\
\hline d X^{\prime}+\omega^{\prime \prime} X^{\prime}-X^{\prime} \omega^{\prime \prime} & 0
\end{array}\right) \\
& \cdots\left(\begin{array}{c|c}
e^{t_{n} X^{\prime \prime} X^{\prime}} & 0 \\
\hline 0 & e^{t_{n} X^{\prime} X^{\prime \prime}}
\end{array}\right) d t_{1} \cdots d t_{n} \text {. }
\end{aligned}
$$

Of course, $\operatorname{ch}(E, \nabla+X)$ is the sum of homogeneous components. Denote the homogeneous component belonging to $\Omega_{Y}^{2 k}$ by $\mathrm{ch}_{k}(E, \nabla+X)$.

The problem of computing character forms of superconnections may be generalized in such a way. Let $\Lambda$ be a graph. Consider a family of representations of $\Lambda$ parameterized by a smooth manifold $Y$. This means a family of vector bundles $E_{\lambda}$ for any vertex $\lambda$ of $\Lambda$ and a family of endomorphisms $E_{\lambda} \rightarrow E_{\mu}$ for any arrow $\lambda \rightarrow \mu$ in $\Lambda$. How can one write down local invariants of such families? The construction of Quillen provides the answer for the graph

We hope to return to this question later.

\section{Variational Complex for Connections}

Here we recall the constructions and facts from [10]. Let $Y$ be a differentiable manifold and $E$ a vector bundle over $Y$. Our first goal is to construct the space of $\infty$-jets of connections in $E$.

Let $F(E)$ be the set of pairs $(y, \omega)$, where $y$ belongs to $Y$ and $\omega$ in an $\infty$-jet of a connection in $E$ at $y$. More precisely, in coordinates, let $U \cong \mathbb{R}^{n}$ be an open subset such that $E \mid U$ is trivialized; let $e_{1}^{U}(y), \ldots, e_{N}^{U}(y)$ be the pointwise basis of $E, y \in Y$. Pick a coordinate system $\left(y^{1}, \ldots, y^{n}\right)$ in $U$. Let $\hat{\Omega}^{1}$ be the standard space of one-forms in variables $y^{1}, \ldots, y^{n}$ whose coefficients are the power series in $y^{1}, \ldots, y^{n}$. Then, for any $y \in U$, the coordinate system gives the isomorphisms $\operatorname{jet}_{y}^{\infty}\left(\Omega_{U}^{1} \stackrel{\sim}{\rightarrow} \hat{\Omega}^{1}\right) ; j^{j e t} t_{y}^{\infty}\left(\Omega \mathcal{1} \otimes g l_{N}\right) \stackrel{\sim}{\rightarrow} \hat{\Omega}^{1} \otimes g l_{N}$. Denote these isomorphisms by $\varphi_{y, U}$. Put $F(E \mid U)=U \times\left(\hat{\Omega}^{1} \otimes g l_{N}(\mathbb{C})\right)$. 
Now, consider the covering of $Y$ by such open sets $U$ that $U \stackrel{\sim}{\rightarrow} \mathbb{R}^{n}$ and $E \mid U$ are trivialized; pick the coordinate systems in the sets $U$; let $e_{i}^{V}(y)=\sum g_{i j}^{U V}(y) e_{j}^{U}(y)$, where $y \in U \cap V$; denote $\infty$-jet of $g^{U V}$ at $y$ by $\bar{g}^{U V}$; for $y \in U \cap V$ and for $\omega \in \hat{\Omega}^{1} \otimes g l_{N}(\mathbb{C})$ put

$$
\psi^{U V}=\varphi_{y, V}\left(d \bar{g}^{U V}\left(\bar{g}^{U V}\right)^{-1}+\bar{g}^{U V} \varphi_{y, U}^{-1}(\omega)\left(\bar{g}^{U V}\right)^{-1}\right) .
$$

By definition

$$
F(E)=\prod_{U} U \times\left(\hat{\Omega}^{1} \otimes g l_{N}(\mathbb{C})\right) / \sim,
$$

where for any $U, V$ and for any $y \in U \cap V, \omega \in \hat{\Omega}^{1} \otimes g l_{N},(y, \omega)_{U} \sim\left(y, \psi^{U V}(\omega)\right)_{V}$.

Denote by $\pi$ the canonical projection $F(E) \rightarrow Y$.

It is clear that $F(E)$ is a projective limit of the finite dimensional manifolds. Thus, one may consider the de Rham complex $\Omega^{*}(F(E))$. Our next goal is to give the canonical decomposition of this complex into horizontal and vertical parts, as mentioned in the Introduction.

To do this, we shall introduce the canonical integrable system of tangent subspaces of $F(E)$. Note first that any connection $\Theta$ in $E$ on an open subset of $Y$ gives a local section of the bundle $F(E) \in \bar{Y}$; an element of the fiber over $y$ corresponding to $\Theta$ is the jet of $\Theta$ at the point $y$. For any $y \in Y$ and any connection $\Theta$ in an open neighborhood of $y$, let $\bar{\Theta}$ be the jet of $\Theta$ at $y$; define the space $V_{y, \Theta}$ as the subspace tangent to the local section of $F(E) \rightarrow Y$ determined by $\Theta$.

\section{Proposition 4.1.}

a) The subspace $V_{y, \Theta}$ depends only on the jet $\bar{\Theta}$ of $\Theta$.

b) The restriction of $\pi_{*}: T_{(y, \bar{\Theta})} F(E) \rightarrow T_{y} Y$ on $V_{y, \boldsymbol{\Theta}}$ is an isomorphism. Thus, $V_{y, \boldsymbol{\theta}}$ is horizontal.

c) The system $\left\{\left(V_{y}, \bar{\Theta}: y, \bar{\Theta}\right) \in F(E)\right\}$ is the integrable distribution.

Proof. All the statements are local in $y$. So, one may check them in coordinates. Under the isomorphism

$$
F(E \mid U) \cong U \times\left(\hat{\Omega}^{1} \otimes g l_{N}\right),
$$

we assume that $y^{1}, \ldots, y^{n}$ are coordinates in $U$ and $y=\left(y_{0}^{1}, \ldots, y_{0}^{n}\right)$; let $(y, \omega) \in F(E \mid U)$; $\omega=\bar{\Theta}$, where $\Theta$ is a $g l_{N}$-valued form on $U ; \Theta=\Sigma \Theta_{i} d y^{i}, \omega=\sum_{i, \alpha} \frac{1}{\alpha !} \partial^{\alpha} \Theta_{i}\left(y-y_{0}\right)^{\alpha} d y^{i}$, where $\alpha$ are multi-indices; so, the space $V_{y, \Theta}$ has the basis $\left(\partial / \partial y^{k} ; \sum_{\alpha, i} \frac{1}{\alpha !} \frac{\partial}{\partial y^{k}}\left(\partial^{\alpha} \Theta_{i}\right)\right.$. $\left.\left(y-y_{0}\right)^{\alpha} d y_{2}^{i}\right), k=1, \ldots, n$; but these vectors are equal to $\left(\partial / \partial y^{k} ; \sum_{\alpha, i} \frac{\partial \omega_{i}^{\alpha}}{\partial y^{k}}\left(y-y_{0}\right)^{\alpha} d y^{i}\right.$ if $\left.\omega=\Sigma \omega_{i}^{\alpha}\left(y-y_{0}\right)^{\alpha} d y_{i}\right)$. This shows that the spaces $V_{y, \Theta}$ are horizontal and depend only on $\omega=\bar{\Theta}$. Moreover, the operators

$$
D_{k}: \omega=\Sigma \omega_{i}^{\alpha}\left(y-y_{0}\right)^{\alpha} d y^{i} \mapsto \Sigma \frac{\partial \omega_{i}^{\alpha}}{\partial y^{k}}\left(y-y_{0}\right)^{\alpha} d y^{i}
$$

commute with each other; thus, the Frobenius integrability condition holds. 
Now, let $\pi$, as before, be the projection $F(E) \rightarrow Y$; let $\pi_{*}$ be the induced map of tangent bundles. We call a tangent vector $\eta$ to $F(E)$ vertical if $\pi_{*} \eta=0$; we call it horizontal if $\eta \in V_{y, \Theta}$ for some $\Theta$. Put

$$
\Omega^{s, t}(F(E))=\left\{\alpha \in \Omega^{s+t}(F(E)): \alpha\left(\eta_{1}, \ldots, \eta_{s_{0}}, \eta_{s_{0}+1}, \ldots, \eta_{s+t}\right)=0\right.
$$

if $\eta_{1}, \ldots, \eta_{s_{0}}$ are horizontal, $\eta_{s_{0}+1}, \ldots, \eta_{s+t}$ are vertical and $\left.s_{0} \neq s\right\}$.

The integrability of our horizontal system gives the decomposition

$$
\Omega^{r}(F(E))=\bigoplus_{s+t=r} \Omega^{s, t}(F(E))
$$

if $\bar{d}$ is the de Rham differential in $\Omega^{*}(F(E))$, then $\bar{d}=d+\delta ; d: \Omega^{s, t} \rightarrow \Omega^{s+1, t} ; \delta: \Omega^{s, t} \rightarrow$ $\Omega^{s, t+1}$.

Now we are going to construct the canonical connection in the vector bundle $\pi^{*} E$ on $F(E)$ which is the pullback of $E$ to the space $F(E)$. In coordinates, assume that we have an open subset $U \subset Y$ and the basis $e_{i}(y)$ as above. Then $F(E \mid U)=\pi^{-1} J_{1}^{-1} U \cong U \times\left(\hat{\Omega}^{1} \otimes g l_{N}\right)$; construct a $g l_{N}$ valued form on $F(E \mid U)$ as follows. Let $(y, \omega) \in U \times\left(\widehat{\Omega}^{1} \otimes g l_{N}\right)$ and $\eta \in T_{(y, \omega)} F(E \mid U)$; then $\pi_{*} \eta \in T_{y} Y$; put

$$
\bar{\omega}(\eta)=\omega\left(\pi_{*} \eta\right)
$$

We get $\bar{\omega} \in \Omega^{1}(F(E \mid U)) \otimes g l_{N}$. It is easy to see that the following statement holds.

Lemma. Let $\{U\}$ be an open covering of $Y$ such that $U \stackrel{\sim}{\rightarrow} \mathbb{R}^{n}$ and $E \mid U$ is trivialized. Then the collection of the forms $\bar{\omega}$ on $F(E \mid U)$ gives a well defined connection in $\pi^{*} E$ on $F(E)$.

Let $\bar{R}_{E}$ be the curvature form of the connection $\bar{\omega} ; \bar{R}_{E}=(d+\delta) \bar{\omega}+\bar{\omega}^{2}$. For any $g l_{N}$-invariant homogeneous polynomial $P$ on the algebra $g l_{N}(\mathbb{C})$ put

$$
\bar{c}_{P}=P\left(\bar{R}_{E}, \ldots, \bar{R}_{E}\right) \in \Omega^{2 m}(F(E)) .
$$

From the above discussion one obtains immediately the following

Theorem 4.1. (cf. [10]). The form $\bar{c}_{P}$ is closed under the differential $d+\delta$ and invariant under the group of automorphisms of $E$.

The theorem implies several identities. For example, let $P(X)=\operatorname{tr} X^{2}$. Then one has:

$$
d \operatorname{tr} \bar{R}_{E}^{2}=0 ; \quad \delta \operatorname{tr} \bar{R}_{E}^{2}=-2 d \operatorname{tr}\left(\bar{R}_{E} \cdot \bar{\omega}\right) ; \quad \delta \operatorname{tr}\left(\bar{R}_{E} \cdot \delta \omega\right)=-\operatorname{tr} d(\delta \omega)^{2} .
$$

The first identity implies that the Chern character of any connection is closed; the second one implies that the cohomology class of this form does not depend on a connection.

\section{Variational Complex for Automorphisms}

Let $Y$ be a smooth manifold and $E$ a vector bundle on $Y$. Put $\widehat{\Omega}^{0}=\mathbb{C}\left[\left[y_{1}, \ldots, y_{n}\right]\right]$;

$$
G(E)=\{(y, \sigma): y \in Y \text {; is an } \infty \text {-jet of an automorphism of } E \text { at } y\} .
$$


In coordinates, if $U$ is a coordinate neighborhood on which $E$ is trivialized then $G(E \mid U)=U \times G L_{N}\left(\hat{\Omega}^{0}\right)$, where $G L_{N}\left(\hat{\Omega}^{0}\right)$ is the space of elements $\sigma \in \hat{\Omega}^{0} \otimes g l_{N}(\mathbb{C})$ such that $\sigma(0) \in G L_{N}$. As before, one easily constructs the horizontal integrable system of tangent subspaces and thus the decomposition

$$
\begin{gathered}
\Omega^{r}(G(E))=\bigoplus_{s+t=r} \Omega^{s, t}(G(E)) ; \\
\bar{d}=d+\delta ; \quad d: \Omega^{s, t} \rightarrow \Omega^{s+1, t} ; \quad \delta: \Omega^{s, t} \rightarrow \Omega^{s, t+1} .
\end{gathered}
$$

Now, let $K(E)=F(E) \underset{Y}{\times} G(E)$ be the fiber product over $Y$. This is the space whose elements are the triples $(y, \omega, \sigma)$, where $w$ is a jet of a connection at $y$ and $\sigma$ is a jet of an automorphism of $E$ at $y$.

Obviously, there is the canonical element $\bar{\sigma}$ of $\Gamma\left(G(E)\right.$, Aut $\left.\pi^{*} E\right) ; \bar{\sigma}(y, \sigma)(e)=\sigma(e)$, where $e \in E_{y}$. Via pullback, we get the connection $\bar{\omega}$ and the automorphism $\bar{\sigma}$ on the space $K(E)$.

Theorem 5.1. The Chern character forms

$$
\overline{\mathrm{ch}}_{k}=\operatorname{ch}\left(\pi^{*} E, \bar{\omega}, \bar{\sigma}\right)
$$

are closed forms on $K(E)$ with respect to the differential $d+\delta$. They are invariant under the group of automorphisms of $E$.

\section{Variational Complex for Superconnections}

Let $Y$ be a smooth manifold and $E=E^{\prime} \oplus E^{\prime \prime}-\mathbb{Z}_{2}$ graded vector bundle over $Y$. Let $F^{s}(E)$ be the space of pairs $(y, \omega)$, where $y \in Y$ and $\omega$ is an $\infty$-jet of a superconnection in $E$ at $y$. In coordinates, let $U$ be an open subset of $Y$ as above; let $\hat{\Omega}^{0}$ be the space of power series in the formal variables $y^{1}, \ldots, y^{n}$; let $N^{\prime}=r k E^{\prime}$, $N^{\prime \prime}=r k E^{\prime \prime}$; then

$$
F^{s}(E \mid U)=U \times\left(\hat{\Omega}^{1} \otimes g l\left(N^{\prime} \mid N^{\prime \prime}\right)^{\mathrm{ev}} \oplus \hat{\Omega}^{0} \otimes g l\left(N^{\prime} \mid N^{\prime \prime}\right)^{\text {odd }} .\right.
$$

The global construction is the straightforward generalization of Sect. 4. We have the projection $F^{s}(E) \stackrel{\pi^{s}}{\longrightarrow} Y$ and also the projection $F^{s}(E) \stackrel{P}{\longrightarrow} F(E)$.

Just the same constructions as before show that there exists the canonical integrable system of tangent subspaces horizontal with respect to $\pi$. This system gives the decompositions

$$
\begin{gathered}
\Omega^{r}\left(F^{s}(E)\right)=\bigoplus_{s+t=r} \Omega^{s, t}\left(F^{s}(E)\right) ; \quad \bar{d}=d+\delta, \\
d: \Omega^{s, t} \rightarrow \Omega^{s+1, t} ; \quad \delta: \Omega^{s, t} \rightarrow \Omega^{s, t+1} .
\end{gathered}
$$

Proceeding as in Sect. 4, we get the following

Theorem 6.1. There exists the canonical superconnection in the vector bundle $\pi^{s *} E$ on $F^{s}(E)$. Its Chern character form $\operatorname{ch}(\bar{\omega}+\bar{X})$ is closed under the differential $d+\delta$ and invariant under the group of automorphisms of $E$. 


\section{Secondary Characteristic Forms}

Let, as above, $Y$ be a smooth manifold, $E$ - a vector bundle over $Y$. Denote by $\xi$ the space of connections in $E$ and $G$-the group $\Gamma(Y$, Aut $E)$ of fibrewise automorphisms of $E$. In Sect. 3, we constructed the Chern character forms $\operatorname{ch}_{k}(E, \nabla) \in \Omega_{Y}^{2 k}$ for any $\nabla \in \xi$ and the forms $\operatorname{ch}_{k}(E, \nabla, \sigma) \in \Omega_{Y}^{2 k-1}$ for any $(\nabla, \sigma) \in \xi \times G$. In this section, for any smooth family $\varphi$ of elements of $\xi \times G$ which is parametrized by an $l$-dimensional manifold $M$, we shall construct the secondary characteristic form $\operatorname{ch}_{k}^{l}(E, \psi) \in \Omega_{Y}^{2 k-1-l}$; similarly, for any smooth $l$-dimensional family $\psi$ of elements of $\xi$ we will construct the forms $\operatorname{ch}_{k}^{l}(E, \psi) \in \Omega_{Y}^{2 k-l}$. If $l=0$ and the family $\varphi$ consists of the single element, these forms $\operatorname{ch}_{k}^{l}(E, \varphi)$ are the Chern character forms. If $l=1$ and $\varphi$ is a path connecting the points $\left(\nabla_{0}, \sigma_{0}\right)$ and $\left(\nabla_{1}, \sigma_{1}\right)$, then $d \operatorname{ch}_{k}^{1}(E, \varphi)=\operatorname{ch}_{k}\left(E, \nabla_{0}, \sigma_{0}\right)-\operatorname{ch}_{k}\left(E, \nabla_{1}, \sigma_{1}\right)$. We will establish similar identities for the higher forms.

Recall from Sects. 4-6 that if $F(E)$ is the variational complex for connections and $G(E)$ is the variational complex for automorphisms, then $F(E)$ and $G(E)$ are the fibrations over $Y$; denote by $K(E)=F(E) \underset{Y}{\times} G(E)$ their fiber product over $Y$, i.e., the space whose points are the triples $(y, \omega, \sigma)$, where $y \in Y, \omega$ is a jet of a connection in $E$ at the point $y$ and $\sigma$ is a jet of an automorphism of $E$ at the point $y$. We denote by $\pi$ the obvious projection $K(E)=F(E) \underset{Y}{\times} G(E) \rightarrow Y$, by $\bar{\nabla}$ and $\bar{\sigma}-$ the universal connection in the bundle $\pi^{*} E$ and the universal automorphism of $\pi^{*} E$ respectively; the differential form $\overline{c h}_{k}=\operatorname{ch}_{k}\left(\pi^{*} E ; \bar{\nabla}, \bar{\sigma}\right)$ belongs to $\Omega^{2 k-1}(K(E))=$ $F(E) \times \underset{Y}{\times} G(E)$; one has the decomposition $\overline{\mathrm{ch}}_{k}=\sum_{l} \overline{\operatorname{ch}}_{k}^{l}$, where $\overline{\operatorname{ch}}_{k}^{l} \in \Omega^{2 k-1-l, l}$. Similarly, $\overline{c h}_{k} \in \Omega^{2 k}(F(E))$ is the universal Chern character form on the variational complex of connections.

Now let $M$ be an $l$-dimensional manifold with boundary and $\varphi: M \rightarrow \xi$ be the smooth map. Construct a form $\operatorname{ch}_{k}^{l}(E, \varphi) \in \Omega_{Y}^{2 k-l}$ as follows. Consider the map $\bar{\varphi}: M \times Y \rightarrow F(E)$, where for $m \in M$ and $y \in Y$,

$$
\bar{\varphi}(m, y)=\left(y, \operatorname{jet}_{y} \varphi(m)\right) \in F(E) .
$$

Consider the pullback $\bar{\varphi}^{*} \overline{\mathrm{ch}}_{k}=\sum_{s} \bar{\varphi}^{*} \overline{\mathrm{ch}}_{k}^{s}$ of the universal Chern character form on $F(E)$. This is the form on $M \times Y$; it is clear that $\bar{\varphi}^{*} \overline{c h}_{k}^{s}$ belongs to $\Omega^{2 k-s}(Y) \otimes \Omega^{s}(M)$ for any s. Put

$$
\operatorname{ch}_{k}^{l}(E ; \varphi)=\int_{M} \bar{\varphi}^{*} \overline{\operatorname{ch}}_{k}^{s} \in \Omega_{Y}^{2 k-l}
$$

Similarly, let $\psi: M \rightarrow \xi \times G$ be a smooth map, where again $M$ is an $l$-dimensional manifold with boundary. Define the map $\bar{\psi}: M \times Y \rightarrow F(E) \underset{Y}{\times} G(E)$ by

$$
\bar{\psi}(m, y)=\left(y, \text { jet }_{y} \psi(m)\right)
$$

and

$$
\operatorname{ch}_{k}^{l}(E ; \psi)=\int_{M} \bar{\psi}^{*} \overline{\operatorname{ch}}_{k}^{s} \in \Omega_{Y}^{2 k-l-1} .
$$

Also, let $E=E^{\prime} \oplus E^{\prime \prime}$ be a $\mathbb{Z}_{2}$-graded bundle, $F^{s}(E)$ - the variational complex for superconnections in $E, \xi^{s}-$ the space of superconnections in $E$. Let $\tau: M \rightarrow \xi^{s}$ be a 
smooth map, $l=\operatorname{dim} M$; define $\bar{\tau}: M \times Y \rightarrow F^{s}(E)$ by

$$
\bar{\tau}(m, y)=\left(y, \text { jet }_{y} \tau(m)\right)
$$

and

$$
\operatorname{ch}_{k}^{l}(E ; \varphi)=\int_{M} \bar{\tau}^{*} \overline{\operatorname{ch}}_{k}^{l} \in \Omega_{Y}^{2 k-l}
$$

These definitions and the fact that $(d+\delta) \overline{\operatorname{ch}}_{k}=0$ imply immediately the following

Theorem 7.1. (i) Let $M$ be an l-dimensional manifold with boundary $\partial M$; let $\mathscr{X}(E)$ be either $\xi$ or $G$ or $\xi$; let $\varphi: M \rightarrow \mathscr{X}(E)$ be a smooth map. Then (i) if $M$ is the point and $\varphi(M)=\nabla$ (respectively $(\nabla, \sigma)$, respectively $(\nabla+X)$, then $\operatorname{ch}_{k}^{0}(E, \varphi)=$ $\operatorname{ch}_{k}\left(E, \nabla\right.$ respectively $\operatorname{ch}_{k}(E, \nabla, \sigma)$ respectively $\left.\left.\operatorname{ch}_{k}(E, \nabla+X)\right)\right)$.

(ii) One has

$$
\operatorname{ch}_{k}^{l-1}(E, \varphi \mid \partial M)=d \operatorname{ch}_{k}^{l}(E, \varphi) \text {. }
$$

Now let us give the explicit formulas for the secondary characteristic forms. We denote by $\Delta^{l}$ the standard simplex $\left\{\left(t_{0}, \ldots, t_{l}\right): t_{i} \geqq 0, \sum t_{i}=1\right\}$. Consider the case of connections. Consider the connections $\nabla_{0}, \ldots, \nabla_{l}$ in the vector bundle $E$ over the smooth manifold $Y$. Assume that in coordinates they are represented by the matrices $\omega_{0}, \ldots, \omega_{l}$. Define the map $\varphi: \Delta^{l} \rightarrow \xi ;\left(t_{0}, \ldots, t_{l}\right) \mapsto \Sigma t_{i} \omega_{i}$. Let $\operatorname{ch}_{k}^{l}\left(E, \omega_{0}, \ldots, \omega_{l}\right)=$ $\operatorname{ch}_{k}^{l}(E, \varphi)$. Then

$$
\begin{aligned}
\operatorname{ch}_{2}^{1}\left(E, \omega_{0}, \omega_{1}\right)= & \frac{1}{2} \operatorname{tr}\left(\operatorname{Ad} A+\frac{2}{3} A^{3}\right), \quad A=\omega_{1}-\omega_{0} ; \\
\operatorname{ch}_{3}^{2}\left(E, \omega_{0}, \omega_{1}, \omega_{2}\right)= & \frac{1}{2} \operatorname{tr}\left(\left(d \omega_{0}+\omega_{0}^{2}+\frac{1}{2}\left(d\left(A_{1}+A_{2}\right)\right.\right.\right. \\
& \left.+\frac{1}{12}\left(A_{1}+A_{2}\right)^{2}+\frac{1}{6} \omega_{0}\left(A_{1}+A_{2}\right)+\frac{1}{6}\left(A_{1}+A_{2}\right) \omega_{0}\right) \\
& \left.\cdot\left(A_{1} A_{2}-A_{2} A_{1}\right)\right), \quad A_{i}=\omega_{i}-\omega_{0} ; \\
\operatorname{ch}_{k}^{k}\left(E, \omega_{0}, \ldots, \omega_{k}\right)= & \frac{1}{k !} \sum_{\sigma \in S_{k}} \operatorname{tr} A_{\sigma 1} \cdots A_{\sigma k} ; \\
\operatorname{ch}_{k}^{l}\left(E, \omega_{0}, \ldots, \omega_{l}\right)= & 0, \quad l>k .
\end{aligned}
$$

These secondary forms give rise to the actions which are the generalizations of the Chern-Simons action from [18]. Let $Y$ be a smooth variety of dimension $2 k$. Fix a connection $\omega_{0}$. Assume that we work in coordinates in which $\omega_{0}$ is represented by zero matrix. Put

$$
Z=\int \exp \left(2 \pi i m \int_{Y} \int_{0}^{1} \frac{1}{(k-1) !} \operatorname{tr} t^{k-1}\left(d A+(t A)^{2}\right)\right) .
$$

Here $m$ is an integer. This is an invariant of the manifold $Y$.

Now, let $Y$ be a smooth manifold, $\omega_{0}, \ldots, \omega_{l}$ - the connections in the vector bundle $E$ over $Y$; let $G=\Gamma(Y$, Aut $E)$ and $\sigma: \Delta^{l} \rightarrow G-$ a smooth map. We write

$$
\left.\operatorname{ch}_{k}^{l}\left(E, \omega_{0}, \ldots, \omega_{l}\right)=\operatorname{ch}_{k}^{l}\left(E, \varphi \mid \omega_{0}, \ldots, \omega_{l}\right) \times \sigma\right),
$$

where, as above, $\varphi\left(\omega_{0}, \ldots, \omega_{l}\right):\left(t_{0}, \ldots, t_{l}\right) \mapsto \sum t_{i} \omega_{i}$. We remind the reader that we denote by $P_{k}$ certain polynomials in two noncommutative variables which have 
been defined in paragraph 2. One has:

$$
\begin{aligned}
\operatorname{ch}_{k}^{l}\left(E, \omega_{0}, \ldots, \omega_{l}, \sigma\right)= & \int_{\Delta_{l}} \operatorname{tr} P_{k}\left(\left(d \sigma+\sum_{0}^{l} \frac{\partial \sigma}{\partial t_{i}} d t_{i}+\left[\sum t_{i} \omega_{i}, \sigma\right]\right) \sigma^{-1} ;(\sigma-1)\right. \\
& \left.\cdot\left(\sum t_{i} d \omega_{i}+d t_{i} \cdot \omega_{i}+\left(\sum t_{i} \omega_{i}\right)^{2}\right)\left(1-\sigma^{-1}\right)\right)
\end{aligned}
$$

In particular:

$$
\operatorname{ch}_{k}^{0}(E, \omega, \sigma)=\operatorname{tr} P_{k}\left((d \sigma+[\omega, \sigma]) \sigma^{-1} ;(\sigma-1) R\left(1-\sigma^{-1}\right)\right)
$$

is the Chern character for $K^{1}$;

$$
\begin{aligned}
& \operatorname{ch}_{k}^{1}\left(E, \omega_{0}, \omega_{1}, \sigma(t)\right) \\
& =\int_{0}^{1} \operatorname{tr} P_{k}\left(\left(d \sigma+\frac{d \sigma}{d t} d t+\left[t\left(\omega_{1}-\omega_{0}\right)+\omega_{0}, \sigma\right]\right) \sigma^{-1} ;(\sigma-1)\left(d t\left(\omega_{1}-\omega_{0}\right)\right.\right. \\
& \left.\left.\quad+d \omega_{0}+t d\left(\omega_{1}-\omega_{0}\right)+\left(\omega_{0}+t\left(\omega_{1}-\omega_{0}\right)\right)^{2}\right)\left(1-\sigma^{-1}\right)\right) .
\end{aligned}
$$

Being written in such a way, this expression depends on a choice of a path $\sigma(t)$. However, for two paths $\sigma_{1}(t), \sigma_{2}(t)$ connecting $\sigma_{0}$ and $\sigma_{1}$, the difference $\operatorname{ch}_{k}^{1}\left(E, \omega_{0}, \omega_{1}, \sigma_{1}(t)\right)-\operatorname{ch}_{k}^{1}\left(E, \omega_{0}, \omega_{1}, \sigma_{0}(t)\right)$ is the closed form representing an integral cohomology class. Thus, one may define the following invariant of an evendimensional manifold. Consider a smooth manifold of dimension $2 k-2$ and a vector bundle $E$ over $Y$. Fix a connection $\omega_{0}$ in $E$. Put

$$
Z=\int D A D \sigma \exp \left(2 \pi i m \int_{Y} \operatorname{ch}_{k}^{1}\left(E, \omega_{0}, A, \sigma(t)\right)\right) .
$$

For $k=2$, this integral appears in the WZW model.

Now, consider the case of superconnections. As before, $\Delta^{l}=\left\{\left(t_{0}, \ldots, t_{l}\right): t_{i} \geqq 0\right.$, $\left.\sum t_{i}=1\right\}$ is the standard complex; having the superconnections $\nabla_{0}+X_{0}, \ldots, \nabla_{l}+X_{l}$, we define $\tau: \Delta^{l} \rightarrow \xi^{s}$ (where $\xi^{s}$ is the space of superconnections in the bundle $\left.E=E^{\prime} \oplus E^{\prime \prime}\right)$ which sends $\left(t_{0}, \ldots, t_{l}\right)$ to $\sum_{0}^{l} t_{i}\left(\nabla_{i}+X_{i}\right)$. We write $\operatorname{ch}_{k}^{l}\left(E, \nabla_{0}+X_{0}, \ldots\right.$, $\left.\nabla_{l}+X_{l}\right)=\operatorname{ch}_{k}^{l}\left(E, \varphi\left(\nabla_{0}+X_{0}, \ldots, \nabla_{l}+X_{l}\right)\right)$. Assume that the connections are represented by the matrices $\omega_{i}$. One has

$$
\begin{aligned}
\operatorname{ch}^{l} & \left(E, \nabla_{0}+\omega_{0}, \ldots, \nabla_{l}+\omega_{l}\right) \\
= & \int_{\Delta^{l}} \sum_{n=0}^{\infty} \operatorname{tr}_{s} \int_{\substack{\text { } \\
0 \leqq s_{0}, \ldots, s_{n} \leqq 0 \\
\sum s_{l}=1}} \exp \left(s_{0}\left(\sum_{0}^{l} t_{i} X_{i}\right)^{2}\right) \\
& \cdot\left(\sum_{0}^{l} d t_{i} \omega_{i}+\sum_{0}^{l} t_{i} d \omega_{i}+\left(\sum_{0}^{l} t_{i} \omega_{i}\right)^{2}+\sum d t_{i} \cdot X_{i}+\sum d X_{i} t_{i}+\left[\sum \omega_{i} t_{i}, \sum X_{j} t_{j}\right]\right)
\end{aligned}
$$




$$
\begin{aligned}
& \left.\left.+(1-t) X_{0}\right]+\left(t d \omega_{1}+(1-t) d \omega_{0}\right)^{2}\right) \cdots \exp \left(s_{p-1}\left(t X_{1}+(1-t) X_{0}\right)^{2}\right) \\
& \cdot\left(X_{1}-X_{0}+d \omega_{1}-d \omega_{0}\right) \cdots \exp \left(s_{n}\left(t X_{1}+(1-t) X_{0}\right) d t d s_{0} \cdots d s_{n}\right. \\
\operatorname{ch}_{1}^{1}( & \left.E, \nabla_{0}+X_{0}, \nabla_{1}+X_{1}\right) \\
= & \operatorname{tr}\left(\left(d X^{\prime \prime}+\omega^{\prime} X^{\prime \prime}-X^{\prime \prime} \omega^{\prime \prime}\right)\left(X^{\prime \prime} X^{\prime}\right)^{-1}\left(e^{X^{\prime \prime} X^{\prime}-1}\right)\right) \\
& -\operatorname{tr}\left(\left(d X^{\prime}+\omega^{\prime \prime} X^{\prime}-X^{\prime} \omega^{\prime}\right)\left(X^{\prime} X^{\prime \prime}\right)^{-1}\left(e^{X^{\prime} X^{\prime \prime}}-1\right)\right)
\end{aligned}
$$

Now, let $Y$ be a smooth manifold and $E=E^{\prime} \oplus E^{\prime \prime}$ a $\mathbb{Z}_{2}$-graded vector bundle over $Y$. Assume that $\operatorname{dim} Y=2 k-1$. Fix a superconnection $\omega_{0}+0$. One may define the integral $(m \in \mathbb{Z})$

$$
Z=\int \exp \left(2 \pi i m \int_{Y} \operatorname{ch}_{k}^{1}\left(E, \omega_{0}+0, A_{1}+X_{1}\right)\right) D A_{1} D X_{1} .
$$

This is an invariant of the manifold $Y$.

\section{The Relation Between Secondary Forms and Primary Forms}

Let $Y$ be a smooth manifold, $E$ a vector bundle over $Y$. Let $\sigma$ be an element of $\Gamma(Y$, Aut $E)$. Let $\nabla$ is a connection in $E$. Then $\nabla^{\sigma}$ is another connection in $E$; it is obtained by changing coordinates in $E$ by means of $\sigma$. In coordinates, if $\nabla=d+\omega$ is covariant derivative with respect to $\nabla$, then $\nabla^{\sigma}=\sigma(d+\omega) \sigma^{-1}=d-d \sigma \cdot \sigma^{-1}+$ $\sigma \omega \sigma^{-1}$ is the covariant derivative with respect to $\nabla^{\sigma}$. Since the characteristic forms $\mathrm{ch}_{m}$ are $\Gamma(Y$, Aut $E)$-invariant, we have $\operatorname{ch}_{m}(E, \nabla)=\mathrm{ch}_{m}\left(E, \nabla^{\sigma}\right)$. Thus, the secondary form $\operatorname{ch}_{m}^{1}\left(E ; \nabla, \nabla^{\sigma}\right)$ is a closed $(2 m-1)$ - form on $Y$.

Theorem 8.1. $\operatorname{ch}_{m}^{1}\left(E ; \nabla, \nabla^{\sigma}\right)=-\operatorname{ch}_{m}(E ; \nabla, \sigma)$.

Proof. Let us assume first that the bundle $E$ and the connection $\nabla$ are trivial. Then $\nabla=d ; \nabla^{\sigma}=d-d \sigma \cdot \sigma^{-1}$; denote $-d \sigma \cdot \sigma^{-1}$ by $A$; then $d A+A^{2}=0$. We have

$$
\begin{aligned}
\operatorname{ch}_{m}^{1}\left(E ; \nabla, \nabla^{\sigma}\right) & =\frac{1}{m !} \int_{0}^{1} \operatorname{tr}\left(d t \cdot A+t \cdot d A+t^{2} A^{2}\right)^{m} \\
& =\frac{1}{(m-1) !} \int_{0}^{1}\left(t^{2}-t\right)^{m-1} d t \cdot \operatorname{tr} A^{2 m-1} \\
& =-\frac{1}{2^{m-1}(2 m-1) ! !} \operatorname{tr}\left(d \sigma \cdot \sigma^{-1}\right)^{2 m-s}=-\operatorname{ch}_{m}(E ; \nabla, \sigma) .
\end{aligned}
$$

This proves the theorem for the special case of trivial bundle and trivial connection. The general case follows as in the proof of Theorem 2.1 .

Theorem 8.1 illustrates the well known topological fact that the Chern character for $K^{1}$ is the transgressed Chern character for $K^{0}$ (or the homotopy equivalence $\Omega B G L(\mathbb{C}) \cong G L(\mathbb{C})$ ). In the next paragraph we shall discuss a more recent and nontrivial example of the same phenomenon. 


\section{Chern Character of Fredholm Modules and Secondary Forms on the Space of Jets of Superconnections}

In this paragraph, we follow essentially Connes and Moscovici [5]. The manifold $Y$ will be now a point. We modify our construction and consider a Hilbert $\mathbb{Z}_{2}$ graded vector bundle over $Y$, i.e., a $\mathbb{Z}_{2}$-graded Hilbert space $E=E_{+} \oplus E_{-}$; a superconnection in $E$ will be a skew selfadjoint operator $X: E \rightarrow E$ which is odd and Fredholm.

Let $A$ be a unital involutive algebra over $\mathbb{C}$ and let $p \in[1, \infty)$. A $p$-summable Fredholm module over $A$ is the followng:

i) a Hilbert space $E=E_{+} \oplus E_{-}$together with an involutive even homomorphism $\rho: A \rightarrow L(E)$, where $L(E)$ is the space of bounded operators;

ii) a skew selfadjoint invertible odd unbounded operator $D$ on $E$ such that $[D, \rho(a)]$ is bounded for any $a \in A$ and $\operatorname{tr}\left(\left(\left(D^{-1}\right)\left(D^{-1}\right)^{*}\right)^{p / 2}\right)<\infty$.

Assume that $(E, D, \rho)$ is a $p$-summable Fredholm module over $A$. Note that the space $F^{s}(E)$ of jets of superconnections coincides with the space of superconnections themselves (because $Y$ is a point). Let $F_{D}^{s}(E)$ be the subspace of operators $D^{\prime}=D+A$, where $A$ is bounded odd and $D^{\prime}$ satisfies ii). Let $\bar{D}$ be the canonical superconnection on $F_{D}^{s}(E)$ - the restriction of that on $F^{s}(E)$.

Connes and Moscovici showed that:

1. the Chern character forms $\operatorname{ch}_{m}(\bar{E}, t \bar{D})$ are well defined on $F_{D}^{s}$ for any $t>0$;

2. $\lim _{t \rightarrow 0} \operatorname{ch}_{n}(\bar{E}, t \bar{D})=0$ if $2 n>p$;

3. $\lim _{t \rightarrow \infty} \operatorname{ch}_{n}(\bar{E}, t \bar{D})=0$;

4. the secondary form $\operatorname{ch}_{n}^{1}(E ; 0, t D)$ is well defined for $t>0$. Moreover, $\lim _{t \rightarrow \infty} \operatorname{ch}_{n}^{1}(E ; 0, t D)$ exists and is a closed $(2 n-1)$ - form on $F_{D}^{s}(E)$.

Here the path between 0 and $t D$ is assumed to be the line segment. The space $F_{D}^{s}$ is equipped with the manifold structure coming from the operator norm of $A, D^{\prime}=D+A$.

5. Let $u$ be a unitary element of $A$, i.e., $u u^{*}=u^{*} u=1$. Then $\gamma_{u}(D)=\rho(u) D \rho\left(u^{*}\right)$ is in $F_{D}^{\mathrm{s}}(E)$. Let $U(A)$ be the group of unitary elements. If we equip $A$ with the norm $\|a\|_{D}=\|\rho(a)\|+\|[D, \rho(a)]\|$, then $U(A) \rightarrow F_{D}^{s}(E)$ is a smooth map.

Pushing back the form $\lim _{t \rightarrow \infty} \operatorname{ch}_{n}^{1}(\bar{E} ; 0, t \bar{D})$, we get a closed $(2 n-1)$-form on $U(A)$.

Moreover, it is invariant under left action of $U(A)$ because the Chern character forms are invariant under coordinate changes.

We can also pass from $A$ to the matrix algebra $M_{N}(A)$ replacing $E$ by $E \otimes \mathbb{C}^{N}, D$ by $D \otimes 1_{\mathbb{C}^{N}}$, ets.; thus, finally we get an invariant closed form on the group $U_{\infty}(A)=\bigcup_{N} U\left(M_{N}(A)\right)$.

The cohomology of the de Rham complex of such forms is well known from $[15,17]$. Recall only that there is the canonical map from $k^{\text {th }}$ cohomology to the cyclic cohomology group $H C^{k-1}(A)$.

Thus, the construction above for each Fredholm module over $A$ provides a family of cyclic cohomology classes in $H C^{2 n+1}(A), n \geqq p / 2$. As shown by Connes and Moscovici, these classes are the Chern characters from [4]. 
We have a strong hope that this interpretation of the Chern character of Fredholm modules, combined with the techniques of [10] and of [8], can be helpful for combinatorial construction of index of elliptic operators.

\section{References}

1. Atiyah, M.: K-Theory. New York: W. A. Benjamin 1967

2. Burghelea, D., Friedlander, L.: Determinants of some elliptic differential operators. Commun. Math. Phys. 138, 1-18 (1991)

3. Berezin, F. A., Retakh, V. S.: A method of computing characteristic classes of vector bundles. Rep. Math. Phys. 18, 363-379 (1980)

4. Chern, S.-S., Simons, J.: Characteristic forms and geometric invariants. Ann. Math., Ser. 2, 99, 48-69 (1974)

5. Connes, A.: Non commutative differential geometry. Publ. Math. I.H.E.S. 62 (1962)

6. Connes, A., Moscovici, H.: Transgression of the Chern character for families and cyclic homology. prepr. I.H.E.S. M/86/33 (1986)

7. Dijkgraaf, R., Witten, E.: Topological gauge theories and group cohomology. Commun. Math. Phys. 129, 393 (1990)

8. Feigin, B., Tsygan, B.: Riemann-Roch theorem and Lie algebra cohomology. In: Proceedings of 2nd Winter School at Srni, Rend. Math. Palermo (1989)

9. Fuks, D. B., Gelfand, I. M., Kazhdan, D.: Actions of infinite dimensional Lie algebras. In: Gelfand, I. M. (ed.). Collected Papers, vol. 3, pp. 349-353. Berlin, Heidelberg, New York: Springer 1987

10. Gabrielov, A. M., Gelfand, I. M., Losik, M. V.: Combinatorial Computation of Characteristic Classes, I, II. In: Gelfand, I. M. (ed.). Collected Papers, vol. 3, pp. 407-436. Berlin, Heidelberg, New York: Springer 1987

11. _ : A local combinatorial formula for the first class of Pontryagin. Ibid. 437-440

12. Gelfand, I. M.: Cohomology of infinite dimensional Lie algebras. Some Questions of Integral Geometry, Report at the International Congress of Mathematicians, Nice, France (1970)

13. Gelfand, I. M., Losik, M. V.: Computing characteristic classes of combinatorial vector bundles. in: Gelfand, I. M. (ed.). Collected Papers, vol. 3, pp.454-486. Berlin, Heidelberg, New York: Springer 1987

14. Karoubi, M.: K-theory. An introduction. Grundlehren, vol. 226. Berlin, Heidelberg, New York: Springer 1978

15. Loday, J. L., Quillen, D.: Cyclic Homology and the Lie Algebra of Matrices. Commun. Math. Helv. 59 (4), 565-591 (1985)

16. Quillen, D.: Superconnections and Chern character. Topology 24, (1) 89-95 (1985)

17. Tsygan, B.: Homology of matrix Lie algebras and Hochschild homology. Uspekhi Mat. Nauk t. 38, 217-218 (1983)

18. Witten, E.: Topological quantum field theory. Commun. Math. Phys. 117, 353-386 (1988)

19. Phys. 121, 351-399 (1989) 